PROCEEDINGS OF THE

AMERICAN MATHEMATICAL SOCIETY

Volume 125, Number 3, March 1997, Pages 717-721

S 0002-9939(97)03983-X

\title{
NOTE ON CLARK'S THEOREM FOR $p$-ADIC CONVERGENCE
}

\author{
MINORU SETOYANAGI
}

(Communicated by Dennis A. Hejhal)

\begin{abstract}
We must read Clark's statement under the hypothesis that the negative of each zero of the indicial polynomial is non-Liouville. In this note we shall give the example for which under the original hypothesis the statement does not hold.
\end{abstract}

\section{INTRODUCTION}

Clark's article [1] is referred to by many authors because he first studied the $p$-adic convergence of power series solutions at a singular point and pointed out the importance of $p$-adic non-Liouvilleness of exponents. However, his result is not properly stated. We must read his statement under the hypothesis that the negative of each zero of the indicial polynomial is non-Liouville. In this note we shall give the example for which under the original hypothesis the statement does not hold. For another approach to $p$-adic convergence using the notion of $p$-adic Liouvilleness in the sense of Schikhof [2], see [3].

Throughout this note $\mathbb{C}_{p}$ denotes the completion of the algebraic closure of $\mathbb{Q}_{p}$, and the $p$-adic valuation ||$_{p}$ is simply written || . By $\mathbb{N}$ we denote the positive integers, and by $\mathbb{N}^{0}$ the non-negative integers. $\mathbb{Z}$ and $\mathbb{Z}_{p}$ denote the rational integers and the ring of $p$-adic integers of $\mathbb{Q}_{p}$, respectively.

\section{Some DEFinitions}

The statement in question is Clark's Theorem 3 [1]. We shall give a summary of this below. To understand it we refer to some definitions in his article.

Definition 1. An element $\alpha \in \mathbb{C}_{p}$ is said to be ( $p$-adically) non-Liouville if for $s \in \mathbb{N}$, we have

$$
\operatorname{ord}(\alpha+s)=O(\log s) \quad \text { as } \quad s \longrightarrow \infty \text {. }
$$

Definition 2. For each non-Liouville number $\alpha$ the weight $w(\alpha)$ is defined by Clark. In this note we only use

$$
w(\alpha)=\frac{1}{p-1}
$$

for $\alpha \in \mathbb{Z}_{p}$.

Received by the editors October 8, 1995.

1991 Mathematics Subject Classification. Primary 12H25; Secondary 11S80, 34G05.

Key words and phrases. p-adic convergence, $p$-adically non-Liouville number.

(C)1997 American Mathematical Society 
Definition 3. The weight $w(g)$ of a polynomial $g \in \mathbb{C}_{p}[X]$ whose roots are nonLiouville is the sum of the weights of the roots.

The ordinal of a polynomial $\Phi \in \mathbb{C}_{p}[X]$, say $\sum_{i=0}^{n} a_{i} X^{i}$, is defined by

$$
\text { ord } \Phi=\min _{i}\left\{\text { ord } a_{i}\right\} \text {. }
$$

(Notice that "minimal" is carelessly mistaken for "maximal" in his article.)

We can summarize Clark's statement as follows:

Consider a linear differential equation

$$
A_{n}(x) y^{(n)}+A_{n-1}(x) y^{(n-1)}+\cdots+A_{0}(x) y=0
$$

where coefficients lie in $\mathbb{C}_{p}[[x]]$ with nonzero radius of convergence. We may write $A_{i}=x^{i} \sum_{j=0}^{\infty} a_{i j} x^{j}(0 \leq i \leq n)$ and $a_{i 0} \neq 0$ for some $i$. Let $\Phi_{j}(s)=\sum_{i=0}^{n} a_{i j}\left(\begin{array}{l}s \\ i\end{array}\right) i$ ! for $j \geq 0$; then $\Phi_{0}$ is the indicial polynomial of (1) at $x=0$.

"Statement". If every zero of the indicial polynomial of (1) is non-Liouville, then each power series solution converges for

$$
\text { ord } x>\sup _{j \geq 1}\left\{\frac{\operatorname{ord} \Phi_{0}-\operatorname{ord} \Phi_{j}}{j}\right\}+w\left(\Phi_{0}\right) .
$$

\section{A NON-LiOUVILLE NUMBeR}

In order to give the example for which the Statement does not hold, we define a special non-Liouville number in the following lemma.

Lemma. Let $\left(e_{k}\right)_{k=1}^{\infty}$ be a sequence of positive integers given by $e_{1}=1$ and $e_{k+1}=$ $k p^{e_{k}}(k \geq 1)$, and let $\lambda=\sum_{k=1}^{\infty} p^{e_{k}}$. Then we have

(i) $\varliminf_{n \rightarrow \infty} \sqrt[n]{|n-\lambda|}=0$;

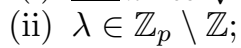

(iii) $\operatorname{ord}(\lambda+n)=O(\log n)$ as $n \longrightarrow \infty$.

Proof. (i) Let $n_{k}=\sum_{j=1}^{k} p^{e_{j}}$. Then $\left(n_{k}\right)_{k=1}^{\infty}$ is a strictly increasing sequence of positive integers, and we have

$$
\sqrt[n_{k}]{\left|\lambda-n_{k}\right|}=p^{-e_{k+1} / n_{k}}
$$

Since $p^{e_{k}} \leq n_{k}<2 p^{e_{k}}$, it follows that

$$
-\frac{e_{k+1}}{n_{k}}<-\frac{e_{k+1}}{2 p^{e_{k}}}=-\frac{k}{2}
$$

and hence

$$
\sqrt[n_{k}]{\left|\lambda-n_{k}\right|}<p^{-\frac{k}{2}}
$$

Thus we have

$$
\lim _{k \rightarrow \infty} \sqrt[n_{k}]{\left|\lambda-n_{k}\right|}=0
$$

and therefore

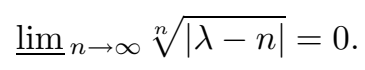


(ii) Clearly $\lambda \in \mathbb{Z}_{p} \backslash \mathbb{N}^{0}$. If $-\lambda \in \mathbb{N}$, that is, there is a positive integer $m$ such that $-\lambda=m$, then we have

$$
\sqrt[n]{|n-\lambda|}=|n+m|^{\frac{1}{n}} \geq\left(\frac{1}{n+m}\right)^{\frac{1}{n+m} \frac{n+m}{n}}
$$

and therefore

$$
\lim _{n \rightarrow \infty} \sqrt[n]{|\lambda-n|}=1
$$

which is contrary to (i).

(iii) Let $n \geq p$, and let

$$
n=a_{0}+a_{1} p+\cdots+a_{l} p^{l}
$$

be the $p$-adic expansion of $n$, where $a_{l} \neq 0$. Then, as $p^{l} \leq n<p^{l+1}$, we have

$$
0 \leq \operatorname{ord}(\lambda+n) \leq l+2 \leq \frac{\log n}{\log p}+2,
$$

and therefore

$$
0 \leq \frac{\operatorname{ord}(\lambda+n)}{\log n} \leq \frac{3}{\log p}
$$

Hence

$$
\operatorname{ord}(\lambda+n)=O(\log n) \quad \text { as } \quad n \longrightarrow \infty .
$$

\section{EXAMPLE}

Now, let $\lambda$ be the non-Liouville number defined above. Consider the linear differential equation

$$
(1-x) x^{2} y^{\prime \prime}+\{(1-\lambda)-(2-\lambda) x\} x y^{\prime}+\lambda x y=0 .
$$

In this equation, we have

$$
\begin{aligned}
& A_{2}=x^{2}(1-x), \\
& A_{1}=x\{(1-\lambda)-(2-\lambda) x\}, \\
& A_{0}=\lambda x,
\end{aligned}
$$

and so

$$
\begin{array}{ll}
a_{20}=1, & a_{21}=-1, \\
a_{10}=1-\lambda, & a_{11}=-(2-\lambda), \\
a_{00}=0, & a_{01}=\lambda, \\
a_{i j}=0 \quad \text { if } j \geq 2 . &
\end{array}
$$

As $\Phi_{j}(s)=a_{0 j}+a_{1 j} s+a_{2 j} s(s-1)$, we have

$$
\begin{aligned}
& \Phi_{0}(s)=(1-\lambda) s+s(s-1)=s(s-\lambda), \\
& \Phi_{1}(s)=\lambda-(2-\lambda) s-s(s-1)=-s^{2}-(1-\lambda) s+\lambda, \\
& \Phi_{j}(s)=0 \quad \text { if } j \geq 2 .
\end{aligned}
$$


The roots of the indicial equation are 0 and $\lambda$, and so the requirement of the Statement is satisfied. Since ord $\lambda \geq 0$ by Lemma (ii), we have

$$
\begin{aligned}
& \operatorname{ord} \Phi_{0}=\min \{\operatorname{ord} 1, \operatorname{ord} \lambda\}=0, \\
& \operatorname{ord} \Phi_{1}=\min \{\operatorname{ord} 1, \operatorname{ord}(1-\lambda), \text { ord } \lambda\}=0, \\
& \operatorname{ord} \Phi_{j}=\infty \text { if } j \geq 2 .
\end{aligned}
$$

This gives

$$
\begin{aligned}
\sup _{j \geq 1}\left\{\frac{\operatorname{ord} \Phi_{0}-\operatorname{ord} \Phi_{j}}{j}\right\}+w\left(\Phi_{0}\right) & =\sup _{j \geq 1}\left\{\frac{-\operatorname{ord} \Phi_{j}}{j}\right\}+w(0)+w(\lambda) \\
& =\frac{2}{p-1} .
\end{aligned}
$$

According to the Statement, the power series solution of (2) must converge for

$$
\text { ord } x>\frac{2}{p-1},
$$

but the equation (2) has the power series solution

$$
y=\sum_{n=0}^{\infty} \frac{x^{n}}{n-\lambda},
$$

whose radius of convergence is zero by virtue of Lemma (i).

\section{4. "COROLlary"}

Clark's original proof of Theorem 3 needs the Corollary on p.266 of his article:

"Corollary". Let $h$ be a monic polynomial whose roots are non-Liouville and in $\left\{\alpha \in \mathbb{C}_{p}:|\alpha| \leq 1\right\}$, and let $t$ be a fixed positive integer greater than all roots of $h$ in $\mathbb{Z}$. If $s, s^{\prime} \in \mathbb{Z}, s>s^{\prime}>t$, then

$$
\sum_{j=s^{\prime}}^{s} \text { ord } h(j)=w(h)\left(s-s^{\prime}\right)+O(\log s) .
$$

Professor Dwork suggested to the author that a similar error appears in this statement. Indeed, if it were true we should have the following claim, which is contrary to our Lemma.

Claim. Let $\alpha \in \mathbb{Z}_{p} \backslash \mathbb{Z}$ such that $\operatorname{ord}(\alpha+s)=O(\log s)$. Then

$$
\underline{\lim }_{s \rightarrow \infty} \sqrt[s]{|s-\alpha|} \geq p^{-\frac{1}{p-1}}
$$

In fact, let $h(X)=X-\alpha$. This is the monic polynomial whose root is only $\alpha$. Therefore

$$
w(h)=w(\alpha)=\frac{1}{p-1} .
$$

By the Corollary we have

$$
\sum_{j=s^{\prime}}^{s} \operatorname{ord}(j-\alpha)=\frac{s-s^{\prime}}{p-1}+O(\log s),
$$


where $s>s^{\prime}>t$. Thus

$$
0 \leq \operatorname{ord}(s-\alpha) \leq \sum_{j=s^{\prime}}^{s} \operatorname{ord}(j-\alpha) \leq \frac{s-t}{p-1}+M \log s
$$

for some $M>0$, so that

$$
|s-\alpha| \geq p^{-\frac{s-t}{p-1}-M \log s},
$$

and therefore

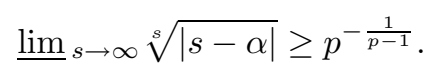

However, this claim contradicts our Lemma when $\alpha=\lambda$.

\section{ACKNOWLEDGEMENT}

The author would like to thank Professor Dwork for his valuable comments and kind advice.

\section{REFERENCES}

1. D. N. Clark, A note on the p-adic convergence of solutions of linear differential equations, Proc. Amer. Math. Soc. 17 (1966), 262-269. MR 32:4350

2. W. Schikhof, Ultrametric calculus, Cambridge Univ. Press, Cambridge, 1984. MR 86j:11104

3. M. Setoyanagi, On the convergence of solutions of $p$-adic linear differential equations, preprint.

Maizuru National College of Technology, 234 Shiraya, Maizuru, Kyoto 625, Japan

E-mail address: set@maizuru-ct.ac.jp 